Original Research Article

\title{
Incidence of methicillin-resistant Staphylococcus aureus (MRSA) infection among patients and hospital staff and impact of preventive measures in reduction of MRSA infection rate: a prospective observational study
}

\author{
Gita Nadimpalli $^{1}{ }^{*}$, Sunil Bhamare ${ }^{2}$, Rao N. P. ${ }^{1}$, Shahu Ingole ${ }^{3}$
}

\begin{abstract}
${ }^{1}$ Department of Internal
Medicine, Rao Nursing Home,

Pune, Maharashtra, India

${ }^{2}$ B.J. Medical College, Pune,

Maharaahtra, India

${ }^{3}$ Department of Medical Affairs,

Emcure Pharmaceuticals, Pune,

Maharashtra, India

Received: 30 September 2016

Accepted: 14 August 2016

*Correspondence to:

Dr. Gita Nadimpalli,

Email:dr.ngita@rnhpune.com

Copyright: (C) the author(s), publisher and licensee Medip Academy. This is an openaccess article distributed under the terms of the Creative Commons Attribution NonCommercial License, which permits unrestricted noncommercial use, distribution, and reproduction in any medium, provided the original work is properly cited.
\end{abstract}

\begin{abstract}
Background: Methicillin-resistant Staphylococcus aureus (MRSA) is associated with difficult to treat infections and high levels of morbidity. It is importance to assess the effectiveness of surveillance screening programs in prevention of MRSA infection. The objective of the study was to assess the type, pattern and antimicrobial sensitivity of MRSA infection and analyse the effectiveness of preventive measures in reduction of MRSA infection rate from 2014 to 2015.

Methods: 1044 and 996 samples with positive cultures obtained from patients admitted in 2014 and 2015 respectively were screened for MRSA using chrome agar test. Only MRSA positive cultures were included in the study and their sensitivity to antibiotics was tested. Screening of MRSA infection was conducted in patients as well as staff of Rao Nursing home, Pune for early identification of MRSA infection and prevention of transmission. MRSA infection rates in 2014 and 2015 were compared.

Results: Community acquired MRSA (CA-MRSA) was more common when compared to Hospital acquired pneumonia (HA-MRSA). HA-MRSA was more common in patients admitted in isolation units. There was a decrease in number of MRSA positive cultures from 2014 (4.8\%) to 2015 (1.3\%), proving the effectiveness of screening for MRSA infection amongst patients as well as healthcare workers. Higher hand hygiene rates were observed in 2015 (95.83\%), which further contributed to the decrease in incidence of MRSA infection in 2015.

Conclusions: Strict adherence to preventive measures of MRSA such as hand hygiene, monitoring and adherence to the bundles for prevention with judicious use of antibiotics can greatly reduce the incidence of MRSA infection.
\end{abstract}

Keywords: Methicillin resistant Staphylococcus aureus (MRSA), Preventive measures, Hand hygiene, Antimicrobial sensitivity

\section{INTRODUCTION}

Methicillin-resistant Staphylococcus aureus (MRSA) infection is caused by a bacteria which has developed resistance to majority of the antibiotics used to treat ordinary staphylococcus infections, which can cause severe problems such as bloodstream infections, pneumonia and surgical site infections in a healthcare setting. ${ }^{1}$ The commonest mode of infection is by direct contact with an infected wound or from contaminated hands, usually those of healthcare providers. Carriers of
MRSA with absence of signs of infection can also spread the bacteria to others and potentially cause an infection. ${ }^{2}$ MRSA infections are commonly acquired in hospitals or other health care settings, such as nursing homes and dialysis centers, and are named as health care-associated MRSA (HA-MRSA) which are associated with use of invasive procedures or devices, such as surgeries, intravenous tubing or artificial joints. ${ }^{3}$ Whereas, another type of MRSA, community-associated MRSA (CAMRSA) is observed in wider community among healthy people, starting as a painful skin boil, and spreading by 
skin-to-skin contact. At-risk populations include groups such as school population, child care workers and people who live in crowded conditions. ${ }^{3}$

Compromised immune system is the primary risk factor for MRSA infection in an in-patient setting. ${ }^{4}$ Apart from it, other population at high risk include infants, the elderly, the chronically ill, burn survivors, organ transplants recipients, cancer patients receiving chemotherapy agents, steroid users, diabetic patients, intravenous drug users, and those with AIDS are the population at high risk of acquiring MRSA infection. Risk factors for HA-MRSA infection include: prolonged length of stay in hospital, high exposure to antibiotics, and exposure to people infected with MRSA. ${ }^{4}$ A previous study has shown that African-American patients and patients with increased lengths of hospital stay are at increased risk of developing MRSA infection, and that female patients had a decreased risk. ${ }^{5}$ In the outpatient or community setting, risk factors for CA-MRSA infection include exposure to an individual with MRSA, usually skin-to-skin contact, and exposure to environments favourable to crowding or a lack of cleanliness. ${ }^{4}$ Hand hygiene has always been the key factor for prevention of infection and limiting the spread of multi-drug resistant organisms (MDROs) as well as susceptible pathogens. Recent studies have reported higher rates of compliance (up to $77 \%$ ) with hand hygiene as compared to less than $40 \%$ on average (ranging from $30-60 \%$ depending on healthcare worker type and unit) in older studies. ${ }^{6}$ However; additional work in this area is needed. Treatment options for MRSA are limited and less effective than options available for susceptible $S$. aureus infections and result in higher morbidity and mortality. However, active screening for MRSA and decolonization in ICU settings is associated with a decrease in MRSA infections, mortality and medical cost as seen in a study. ${ }^{7}$

Hence, this study was conducted to assess the effectiveness of preventive measures like hand hygiene and screening of MRSA in patients as well as healthcare workers in reducing the incidence of MRSA.

\section{METHODS}

After obtaining Independent Ethics Committee approval and taking written informed consent of patients and healthcare workers of Rao Nursing Home, Pune, 1044 samples with positive cultures from patients admitted in 2014 and 996 samples with positive cultures from patients admitted in 2015 were screened for MRSA using chrome agar test. The swabs for culture were collected from nose and groin and plated on the chrome agar plate to check for color change, after which they were tested in the Microbiology department at Rao Nursing Home using (Mannitol sugar and coagulase test). All cases where swab was positive for MRSA nasal/ groins were considered as positive for MRSA and were included in the study. All other strains such as Methicillin sensitive Staphylococcus aureus (MSSA) were excluded. The data was analyzed under the following headings: i) Comparison of percentage positivity of samples of MRSA of 2014 and 2015, ii) type of sample in which positive culture sensitivity was recorded, iii) distribution pattern of MRSA positive cases in hospital during 2014 and 2015, iv) comparison of HAMRSA cases against CAMRSA, v) antibiotic sensitivity pattern of 2014 cases versus 2015 cases, vi) device utilization rates relationship with MRSA infection, vii) hand hygiene rates and their relation to MRSA, and viii) screening of healthcare workers for MRSA. All cases of laboratory confirmed MRSA were analyzed for 2015 against the data of 2014. Screening of staff for MRSA was carried out as part of patient and staff safety programme of staff working in critical care areas. Data was analyzed using descriptive statistics like number and percentages.

\section{RESULTS}

Table 1: Comparison-percentage positivity of samples of MRSA of 2014 and 2015.

\begin{tabular}{|lll|}
\hline Sample Details & $\mathbf{2 0 1 4}$ & $\mathbf{2 0 1 5}$ \\
\hline Total No. of microbiology samples & 6234 & 8013 \\
\hline Total no of positive cultures & 1044 & 996 \\
\hline Total no. of MRSA positive cultures & 50 & 13 \\
\hline $\begin{array}{l}\text { \% of positive cases of MRSA of total } \\
\text { positive cases }\end{array}$ & $4.8 \%$ & $1.3 \%$ \\
\hline In patients day & 27933 & 23683 \\
\hline MRSA Infection incidence rate & 0.17 & 0.054 \\
\hline
\end{tabular}

Table 2: Type of sample in which positive c/s was recorded.

\begin{tabular}{|lll|}
\hline Type of sample & $\begin{array}{l}\mathbf{2 0 1 4}(\mathbf{N}=\mathbf{5 0}) \\
\mathbf{n}(\%)\end{array}$ & $\begin{array}{l}\mathbf{2 0 1 5}(\mathbf{N}=\mathbf{1 3}) \\
\mathbf{n}(\%)\end{array}$ \\
\hline Urine & $5(10 \%)$ & $0(0 \%)$ \\
\hline Tracheostomy & $1(2 \%)$ & $0(0 \%)$ \\
\hline BAL & $1(2 \%)$ & $0(0 \%)$ \\
\hline Blood & $7(14 \%)$ & $0(0 \%)$ \\
\hline ETT & $1(2 \%)$ & $1(7.7 \%)$ \\
\hline Swab & $1(2 \%)$ & $0(0 \%)$ \\
\hline Oral Secretions & $1(2 \%)$ & $0(0 \%)$ \\
\hline PUS & $4(8 \%)$ & $0(0 \%)$ \\
\hline PUS Swab & $24(48 \%)$ & $12(92.3 \%)$ \\
\hline Sputum & $3(6 \%)$ & $0(0 \%)$ \\
\hline Wound Swab & $2(4 \%)$ & $0(0 \%)$ \\
\hline
\end{tabular}

Table 3: Distribution pattern of MRSA positive cases in hospital 2014 and 2015.

\begin{tabular}{|lll|}
\hline Location & $\mathbf{2 0 1 4}(\mathbf{N}=\mathbf{5 0}) \mathbf{n}(\boldsymbol{\%})$ & $\mathbf{2 0 1 5}(\mathbf{N}=\mathbf{1 3}) \mathbf{n}(\boldsymbol{\%})$ \\
\hline ICU & $\mathbf{9}(18 \%)$ & $3(23 \%)$ \\
\hline Cay Care & $1(2 \%)$ & $0(0 \%)$ \\
\hline Isolation & $14(28 \%)$ & $6(46 \%)$ \\
\hline MGW & $8(16 \%)$ & $2(15.5 \%)$ \\
\hline OPD & $11(22 \%)$ & $2(15.5 \%)$ \\
\hline Rose & $7(14 \%)$ & $0(0 \%)$ \\
\hline
\end{tabular}


Out of the total number of positive cultures in 2014 (1044), about $4.8 \%$ of samples were MRSA positive, the number was observed to decreased in 2015 , only $1.3 \%$ of the total number of positive culture in 2015 (996) were tested to be MRSA positive (Table 1). Pus swab was most commonly used for collection of sample for culture in 2014 (48\%) and 2015 (92.3\%) (Table 2). Maximum number of MRSA positive cultures for hospital acquired MRSA were from samples obtained from isolation rooms, $28 \%$ and $46 \%$ for the years 2014 and 2015 respectively (Table 3). Numerically, the number of hospital acquired MRSA was less in 2015 (7.6\%) when compared to $2014(92.4 \%)$ (Table 4). However, the community acquired MRSA was more common when compared to hospital acquired MRSA. Sensitivity of
Tigecycline and Chloramphenicol for treating MRSA declined from 2014 to 2015 (Table 5). There was increase in device utilization rates from 2014 to 2015 (Table 6) with a concomitant increase in hand hygiene rates. Out of 117 staff who were screened for MRSA in 2015, 6 tested positive for MRSA due to the exposure to the patients with MRSA (Table 7).

Table 4: Comparison of HAMRSA cases against CAMRSA.

\begin{tabular}{|lll|}
\hline Year & HA MRSA $\mathbf{n}(\%)$ & CA MRSA $\mathbf{n}(\%)$ \\
\hline $2014(\mathrm{~N}=50)$ & $10(20 \%)$ & $40(80 \%)$ \\
\hline $2015(\mathrm{~N}=13)$ & $1(7.6 \%)$ & $12(92.4 \%)$ \\
\hline
\end{tabular}

Table 5: Antibiotic sensitivity pattern of 2014 cases v/s 2015 cases.

\begin{tabular}{|lllllll|}
\hline MRSA & Vancomycin & Linezolid & Teicoplanin & Tigecycline & Doxycycline & Chloramphenicol \\
\hline Sensitivity 2015 & $100 \%$ & $100 \%$ & $100 \%$ & $92 \%$ & $92 \%$ & $92 \%$ \\
\hline Sensitivity 2014 & $98 \%$ & $100 \%$ & $100 \%$ & $100 \%$ & $66 \%$ & $98 \%$ \\
\hline
\end{tabular}

Table 6: Device utilization rates relation with MRSA infection.

\begin{tabular}{|lll|}
\hline DUR & $\mathbf{2 0 1 4}$ & $\mathbf{2 0 1 5}$ \\
\hline ETT $^{*}$ & 32.04 & 38.88 \\
\hline UC* $^{*}$ & 158.04 & 221.4 \\
\hline CL $^{*}$ & 129.48 & 153.36 \\
\hline HD $^{*}$ & 9.48 & 45.24 \\
\hline
\end{tabular}

*ETT-Endotracheal tube, UC-Urinary catheter, CLCentral line, HD-Hemodialysis

Table 7: Comparison of hand hygiene rates.

\begin{tabular}{|ll|}
\hline Hand hygiene rates \\
\hline 2014 & 2015 \\
\hline $88.50 \%$ & $95.83 \%$ \\
\hline
\end{tabular}

Table 8: Screening of health care workers for MRSA.

\begin{tabular}{|lll|}
\hline Year & $\mathbf{2 0 1 4}$ & $\mathbf{2 0 1 5}$ \\
\hline No. of staff screened & 84 & 117 \\
\hline No. of staff positive in screening & Nil & 6 \\
\hline
\end{tabular}

\section{DISCUSSION}

It was encouraging to see that the number of MRSA positive samples had decreased from 2014 to 2015, indicating better preventive measures and awareness of MRSA. It was similar to a previous study, where improved MRSA infection rates have been demonstrated in certain patient populations where more aggressive measures were taken than standard barrier and isolation practices. $^{8}$ Observing a substantial number of MRSA positive cultures in 2014, screening of patients for MRSA was conducted in 2015 to avoid missing of positive cases. A previous study has depicted that active screening for MRSA and decolonization, especially in ICU settings with a high MRSA infection rate is associated with a decrease in MRSA infections, mortality and medical cost. ${ }^{7}$ Our study also suggests that surveillance programs are effective in decreasing these infections. In 2014, the maximum positive samples were recorded in pus followed by blood, urine and sputum, whereas the majority of the positive samples in 2015 were of pus. Hence, pus can be considered as a good sample to test for MRSA infection as seen in a previous study. ${ }^{9}$

The incidence of CA-MRSA was more as compared to HA-MRSA, similar findings were observed in a previous study. ${ }^{10}$ However, most of these CA-MRSA infections involve skin and soft tissue types, which respond quickly to wound care (incision and drainage) and outpatient oral antimicrobial therapy. ${ }^{10}$ In addition, patients with CAMRSA infections have absence of risk factors associated with HA-MRSA infections, which include recent hospitalization, dialysis, nursing-home residence, and other co-morbid conditions such as diabetes, chronic renal failure, and chronic pulmonary diseases which bring them into contact with healthcare settings. Assessment of pattern of MRSA positive cases in hospital in 2014 depicted that the maximum cases of HA-MRSA were recorded in isolation units which was consistent in 2015. Owing to the increasing incidence of healthcareassociated infections (HAI), HAI has been identified as a focus area for achieving the goal of healthy people 2020, with reduction of invasive healthcare-associated MRSA infections named as a top priority. ${ }^{11}$ Decreased sensitivity of MRSA to Tigecycline was observed which could be probably due to non-judicious use of drug, change in 
strains of organisms and hence the sales of antibiotic recorded for Tigecycline was decreased to 121 in 2015 when compared to 159 in 2014.

Though the device utilization rates (DUR) were increased in 2015 in comparison to 2014, there was a decrease in number of cases of MRSA due to strict implementation of Bundles used to prevent HAI infections, along with better hand hygiene rates as observed amongst the healthcare workers in 2015 when compared to 2014. However, hand hygiene alone cannot singularly inhibit the influence of formidable risk factors such as HAI acquisition at an older age, admission to the ICU, length of stay longer than average, or the fourfold increased risk of infection in patients colonized with $S$. aureus. ${ }^{12}$ Additional measures like decolonization of MRSA carriers, enhanced cleaning of healthcare equipment and hospital-wide culture change program for 3 years have proved to be effective in controlling endemic MRSA infection in other countries. ${ }^{13}$

Screening of staff for MRSA was carried out as part of patient and staff safety programme in critical care areas. In 2014, staff screened did not record positive cases, however, with admission of cases of MRSA positive in 2014, the staff were also exposed to the organisms and got colonized, which were screened positive for MRSA in 2015 and were decolonized to control the spread of disease. In one study, it was observed that MRSA infection rates decreased by $93 \%$ in cardiac surgical wounds after a program was initiated that not only screened patients, but also included additional interventions such as decolonizing hospital staff who screen positive, providing vancomycin prophylaxis for patients who screen positive, and administering mupirocin calcium nasal ointment for all patients regardless of screening status. ${ }^{14}$

Strict implementation of preventive measures such as hand hygiene, monitoring of device utilization and adherence to the bundles for prevention of healthcare associated infection are detrimental in reducing MRSA infection rate. Medical practitioners can play a crucial role in identification and control of MRSA infections by active screening of patients for MRSA, contact isolation of patients, and patients and healthcare staff education.

\section{ACKNOWLEDGEMENTS}

The authors thank the entire staff of Rao nursing home for help in collection of data, and all the patients for their cooperation.

\section{Funding: No funding sources} Conflict of interest: None declared

Ethical approval: The study was approved by the Institutional Ethics Committee

\section{REFERENCES}

1. General Information about MRSA in the Community [Internet]. Centers for Disease Control and Prevention. Centers for Disease Control and Prevention; 2016 [cited 2016Sep26]. Available from: http://www.cdc.gov/mrsa/community/

2. Mccarley GA, Turkel Stephanie. Methicillin-resistant Staphylococcus aureus (MRSA) Infections Essay [Internet]. Methicillin-resistant Staphylococcus aureus (MRSA) Infections Essay. [cited 2016Sep26]. Available from: http://123student.com/essays/methicillin-resistantstaphylococcus-aureus-mrsa-infections_1

3. MRSA infection- Mayo Clinic. 2015 [cited 2016Sep26]. Available from: http://www.mayoclinic.org/diseasesconditions/mrsa/basics/definition/con-20024479

4. Green BN, Johnson CD, Egan JT, Rosenthal M, Griffith EA, Evans MW. Methicillin-resistant Staphylococcus aureus: an overview for manual therapists. Journal of Chiropractic Medicine. 2012;11(1):64-76.

5. Jennings A, Bennett M, Fisher T, Cook A. Impact of a surveillance screening program on rates of methicillin-resistant Staphylococcus aureus infections with a comparison of surgical versus nonsurgical patients. Baylor University Medical Center Proceedings. 2014;27(2):83-7.

6. Barnes SL, Morgan DJ, Harris AD, Carling PC, Thom KA. Preventing the Transmission of Multidrug-Resistant Organisms: Modeling the Relative Importance of Hand Hygiene and Environmental Cleaning Interventions. Infect Control Hosp Epidemiol Infection Control and Hospital Epidemiology. 2014;35(09):1156-62.

7. Lee YJ, Chen JZ, Lin HC, Liu HY, Lin SY, Lin HH, et al. Impact of active screening for methicillinresistant Staphylococcus aureus (MRSA) and decolonization on MRSA infections, mortality and medical cost: a quasi-experimental study in surgical intensive care unit. Critical Care. 2015;19(1):143.

8. Hawkins House, Hawkins Street, Dublin, Ireland. Prevention and Control Methicillin-Resistant Staphylococcus aureus (MRSA). Prevention and Control Methicillin-Resistant Staphylococcus aureus (MRSA) National clinical effectiveness committee; Dec, 2013. Available from: http://www.hpsc.ie/AZ/MicrobiologyAntimicrobialResistance/InfectionCo ntrolandHAI/Guidelines/File, 14478,en.pdf

9. Khan MF, Neral A, Yadav VC, Khan FA, Ahamed S. Incidence and antibiotic susceptibility of methicillin resistant Staphylococcus aureus in pus samples of patients of Bastar region, Central India. South Asian J Exp Biol. 2012;3(5):2014-8.

10. Huang H, Flynn NM, King JH, Monchaud C, Morita M, Cohen SH. Comparisons of CommunityAssociated Methicillin-Resistant Staphylococcus aureus (MRSA) and Hospital-Associated MSRA 
Infections in Sacramento, California. J. CLIN. MICROBIOL. 2006;44(7):2423-7.

11. Gidengil CA, Gay C, Huang SS, Platt R, Yokoe D, Lee GM. Cost-Effectiveness of Strategies to Prevent Methicillin-Resistant Staphylococcus aureus Transmission and Infection in an Intensive Care Unit. Infect Control Hosp Epidemiol Infection Control and Hospital Epidemiology. 2015;36(01):17-27.

12. Mclawas ML. The relationship between hand hygiene and health care-associated infection: it's complicated. Infection and Drug Resistance. 2015;8:7-18.

13. Marimuthu K, Pittet D, Harbarth S. The effect of improved hand hygiene on nosocomial MRSA control. Antimicrob Resist Infect Control
Antimicrobial Resistance and Infection Control. 2014;3(1):34

14. Walsh EE, Greene L, Kirshner R. Sustained reduction in methicillin resistant Staphylococcus aureus wound infections after cardiothoracic surgery. Arch Intern Med. 2011;171(1):68-73.

Cite this article as: Nadimpalli G, Bhamare S, Rao $\mathrm{NP}$, Ingole $\mathrm{S}$. Incidence of methicillin-resistant Staphylococcus aureus (MRSA) infection among patients and hospital staff and impact of preventive measures in reduction of MRSA infection rate: a prospective observational study. Int J Basic Clin Pharmacol 2016;5:2336-40. 